\title{
Efecto de la psicoeducación en el afrontamiento y adaptación al rol de cuidador familiar del adulto mayor
}

\section{Effect of psychoeducation in the support of the older adult family caregiver adaptation and coping role}

\section{Efeito da psicoeducação no enfrentamento e adaptação ao papel de cuidador familiar do idoso}

\section{J.J. Cuevas-Cancino ${ }^{\mathrm{at}}{ }^{*}$, N.E. Moreno-Pérez ${ }^{\mathrm{b} 1}$, M.J. Jiménez-González ${ }^{\mathrm{c}}$, N. Padilla-Raygoza ${ }^{\mathrm{d} 1}$, I. Pérez-Zamora ${ }^{\mathrm{e} 2}$, L. Flores-Padilla ${ }^{\mathrm{f}_{3}}$}

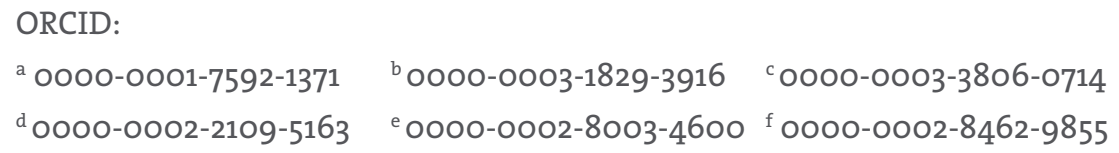

${ }^{1}$ Departamento de Enfermería Clínica, División de Ciencias de la Salud e Ingenierías, Campus Celaya Salvatierra, Universidad de Guanajuato, Guanajuato, México ${ }^{2}$ Coordinación Clínica de Educación e Investigación, UMF 6o, Delegación Regional Estado de México Oriente, Instituto Mexicano del Seguro Social, Estado de México, México ${ }_{3}^{3}$ Unidad de Investigación en Epidemiologia y Servicios de Salud, Hospital Regional de Zona \# 6, Instituto Mexicano del Seguro Social, Ciudad Juárez, Chihuahua, México

Recibido: 6 marzo 2019/Aceptado: 26 julio 2019 
en la longevidad; los individuos viven más, pero con un bienestar deficiente debido a las limitaciones y comorbilidades inherentes al envejecimiento, con las que a menudo necesitan el cuidado de alguien. Actualmente, uno de cada cuatro adultos mayores requiere de ayuda para realizar sus actividades de la vida diaria.

Objetivo: Evaluar el efecto de una intervención de enfermería por medio de la psicoeducación para facilitar el proceso de afrontamiento y adaptación al rol de cuidador familiar del adulto mayor.

Material y Métodos: Estudio cuantitativo, cuasi experimental, realizado en una unidad de medicina familiar, con un grupo de 70 cuidadores familiares de adultos mayores, los criterios de inclusión: adultos de 20 a 59 años que supieran leer y escribir. La intervención psicoeducativa consistió en 10 sesiones, los instrumentos aplicados pre y post intervención fueron la escala sobre el proceso de afrontamiento y adaptación, así como la que evalúa habilidad de cuidado. Se utilizó estadística descriptiva e inferencial.

Resultados: La edad de los cuidadores familiares tuvo una media de 50.6, femeninos 92.8\%. La intervención fue efectiva, pues se obtuvieron diferencias estadísticamente significativas entre el pre y post en afrontamiento y adaptación ( $\mathrm{X}^{2} \mathrm{Mc}$ nemar $\left.\mathrm{p}=0.00001\right)$, así como para la habilidad de cuidado $\left(\mathrm{X}^{2} \mathrm{p}=0.01\right)$, lo que sugiere un efecto positivo y sostenido de la intervención.

Conclusiones: Los cuidadores familiares de los adultos mayores que participaron en la intervención, mostraron un efecto positivo en la adaptación a su rol de cuidador familiar.

Palabras clave: Psicoeducación; cuidador familiar; habilidad de cuidado; adaptación psicológica; México.

\section{ABSTRACT}

Introduction: The present demographic transition, influenced by science and technology advances aimed at improving the quality of life, features a greater longevity; however, older adults sometimes suffer from limitations and comorbidities which are related to their aging process and which make them require caring assistance. Currently, around $25 \%$ of older adults require some kind of aid in order to perform their daily living activities.

Objective: To assess the effect of a nursing intervention using psychoeducation to support the older adult family caregiver adaptation and coping role.

Methods and materials: This is a quantitative quasi-experimental study carried out in a unit of family medicine with 70 family caregivers of older adults. The inclusion criteria was: adults aged 20 to 59 who could read and write. The intervention included 10 sessions. The pre and post applied instruments were the Coping Adaptation Processing Scale, and a scale to assess the ability to provide care. Descriptive and inferential statistics were calculated.

Results: The mean age of the family caregivers was 50.6 years. 65 were female. The intervention turned out to be effective as statistically significant differences in coping and adaptation measures ( $\mathrm{X}^{2} \mathrm{Mc}$ Nemar $\left.\mathrm{p}=0.00001\right)$, as well as caregiving abilities $\left(\mathrm{X}^{2} \mathrm{p}=0.01\right)$ measures, were obtained at the post intervention assessment.

Conclusion: The older adult family caregivers who participated in this nursing intervention showed a positive effect in their role.

Keywords: Psychoeducation; family caregiver; care ability; adaptation, psychological; México 


\section{RESUMO}

Introdução: A transição demográfica e epidemiológica mundial, os avanços na ciência e tecnologia aplicados em melhorar a qualidade de vida da população, influenciam a longevidade; os indivíduos vivem mais, no entanto com um bem-estar deficiente, devido às limitações e comorbilidades inerentes ao envelhecimento, regularmente necessitam o cuidado de alguém. Atualmente, um de cada quatro idosos requerem de ajuda para realizar suas atividades da vida diária.

Objetivo: Avaliar o efeito de uma intervenção de enfermagem através da psicoeducação para facilitar o processo de enfrentamento e adaptação no papel de cuidador familiar do idoso.

Material e Métodos: Estudo quantitativo, quase experimental, realizado na unidade de medicina familiar, com um grupo de 70 cuidadores familiares de idosos, os critérios de inclusão: adultos de 20 a 59 anos, que soubessem ler e escrever. A intervenção psicoeducativa consistiu em 10 sessões, os instrumentos aplicados pre e post intervenção foram a escala sobre o processo de enfrentamento e adaptação, assim como a que avalia habilidade de cuidado. Utilizou-se estatística descritiva e inferencial.

Resultados: A idade dos cuidadores familiares teve una média de 50.6, femininos 92.8\%. A intervenção foi efetiva, pois se obtiveram diferenças estatisticamente significativas entre o pre e post em enfrentamento e adaptação ( $\mathrm{X}^{2} \mathrm{Mc}$ nemar $\left.\mathrm{p}=0.00001\right)$, assim como, para a habilidade de cuidado ( $\mathrm{X}^{2} \mathrm{p}=0.01$ ), o que sugere um efeito positivo e sustenido da intervenção. Conclusões: Os cuidadores familiares dos idosos que participaram na intervenção mostraram um efeito positivo na adaptação do seu papel de cuidador familiar.

Palavras chave: Psicoeducação; cuidador familiar; habilidade de cuidado; adaptação psicológica; México.

\section{INTRODUCCIÓN}

Los avances en la ciencia, así como en la tecnología, aplicados y utilizados en mejorar la calidad de vida de la población, de la salud y de los servicios, aunado al descenso de la natalidad y la mortalidad, se reflejaron en el aumento de la esperanza de vida con tendencia a una transición demográfica en la que la pirámide poblacional se ha invertido². La población mundial llegó a 7000 millones en 2011; a su vez, según estimaciones de la OMS en el año 2010, 524 millones de personas tenían una edad de 65 años o más, lo que equivale al 8\% de la población mundial. Se prevé que en 2050 esta cifra casi se triplicará hasta ser de unos 1500 millones y representará el 16\% de la población mundial 2-5.

El aumento de la esperanza de vida puede considerarse un éxito de las políticas de salud pública y del desarrollo socioeconómico, pero el incremento del grupo de personas mayores genera otras demandas para los servicios de salud con programas específicos de promoción de la salud, prevención de la enfermedad, rehabilitación, cuidados urgentes/crónicos y cuidados paliativos.

En el Instituto Mexicano del Seguro Social (IMSS) se aprecian estos cambios demográficos, ya que el promedio de edad de la población derechohabiente actualmente es 20 años mayor que el de la población general. Este grupo, no sólo está más envejecido, sino que la tasa de crecimiento de los adultos mayores es más elevada que en cualquier otro grupo: en 1990 la proporción de adultos mayores de 60 años fue de 9.2\%; en el 2000 de $12.3 \%$ y en el 2010 de $16.1 \%$; es decir, este grupo de edad se ha duplicado en los últimos 20 años y se estima que llegará a 28\%, para el 2050, lo que representará que uno de cada cuatro derechohabientes será adulto mayor. En contraste, hoy en día del 30 al 50\% de la población hospitalizada es un adulto mayor ${ }^{1,3}$. 
Los individuos viven más, pero con un bienestar deficiente, debido a las limitaciones propias del envejecimiento y sus comorbilidades; para algunos de los adultos mayores (AM) es necesario el cuidado y apoyo de alguien más para desempeñar las actividades de la vida diaria ${ }^{4.5}$, lo que da lugar al surgimiento de la figura del cuidador. Se denomina como tal a las personas que asisten o cuidan a otra persona, por cualquier causa de minusvalía, o incapacidad que le dificulta o impide el desarrollo normal de sus actividades o de sus relaciones ${ }^{6}$.

La persona que asume las funciones o rol de cuidador familiar, presenta una necesidad de adaptación o afrontamiento ante el desempeño de este nuevo rol, un mejor o peor ajuste se logrará según la valoración de esta situación, así como de los recursos y habilidades con que cuente, entendidos como los esfuerzos cognitivos y conductuales en constante cambio, que sirven para manejar las demandas externas e internas, que son valoradas como excedentes o desbordantes de los recursos del individuo, derivadas del cuidado del AM. En otras palabras, puede decir que las formas en que el cuidador familiar afronta las demandas de cuidado dependen de lo estresante de la situación?.

En relación con la pérdida de autonomía del AM, Manrique-Espinoza mostró que uno de cada cuatro AM (26.9\%) exhibe algún grado de limitación para las actividades básicas de la vida diaria. Estos datos muestran que la dependencia funcional es uno de los problemas más graves de los AM, ya que dificulta la atención y el acceso a los servicios de salud y es particularmente disruptiva cuando se acompaña del deterioro cognitivo ${ }^{8}$.

El deterioro del estado de salud de los AM tiene un impacto directo sobre la morbilidad general y la utilización de los servicios de salud; como resultado, los egresos hospitalarios para este grupo poblacional se han acrecentado en los últimos seis años. Los hogares con personas mayores consumen 50\% más recursos en salud que el promedio, y los AM tienen una probabilidad doble de ser hospitalizados con respecto a los más jóvenes9.

A partir de los datos observados queda claro que es necesaria la puesta en marcha de un nuevo modelo de atención para hacer frente a la creciente prevalencia de las enfermedades crónico degenerativas, así como al aumento de la discapacidad y consecuente dependencia que resulta de ella en los adultos mayores. Se requiere, por tanto, no sólo atender a los AM sino a las personas que en el hogar se responsabilizan de su cuidado, de forma que brinden atención pertinente y adecuada, pero lo realicen con el menor desgaste emocional posible.

A este tipo de situación a la que se enfrenta el cuidador familiar puede denominarse afrontamiento, el cual es definido como los modos innatos o adquiridos de actuar de una persona ante los cambios producidos en el entorno, al igual que el conjunto de esfuerzos conductuales y cognitivos que realiza para hacer frente a las situaciones estresantes, esto con el fin de reducir el estado de malestar que le produce.

Por otra parte, el proceso de afrontamiento y adaptación desde la perspectiva del modelo conceptual de Callista Roy, es definido como los patrones innatos o adquiridos de las formas de manejar y responder al ambiente cambiante, en las situaciones de la vida diaria y en los periodos críticos mediante comportamientos directamente enfocados a resolver la situación problema.

Con base en una revisión bibliográfica se eligió la psicoeducación como modelo para implementar una intervención, al ser esta una aproximación terapéutica en la cual se proporciona a los familiares del paciente información específica acerca de la enfermedad. La psicoeducación es un proceso en el cual un facilitador (profesional del área de la salud) detalla y resume información científica actual y relevante para responder preguntas acerca del origen, evolución, así como el abordaje de 
un padecimiento desde su entorno; situación que implica también la consejería y entrenamiento en técnicas para afrontar el problema ${ }^{10}$.

La psicoeducación, ha demostrado ser una forma de abordaje de los problemas de la familia que puede tener importantes beneficios porque potencia el intercambio de experiencias entre personas que están en situaciones similares, y con ello se fomentan las redes de apoyo social informal, pues proporciona un escenario sociocultural de construcción del conocimiento. La psicoeducación aplicada por Enfermería puede ser eficaz, ya que, además de ser otra opción para cuidar al paciente y a la familia, favorece una alianza terapéutica, reconoce el ambiente donde se desarrolla la familia y le ayuda acrecentar sus habilidades necesarias y conductas que fomenten la salud individual y colectiva ${ }^{10}$.

El objetivo del estudio fue evaluar el efecto de una intervención de enfermería a través de psicoeducación para facilitar el proceso de afrontamiento y adaptación, al rol del cuidador familiar del adulto mayor.

\section{MATERIAL Y MÉTODOS}

El tipo de estudio fue cuantitativo, diseño cuasi experimental, en el que participaron un grupo de cuidadores familiares. Se realizaron mediciones pre y post intervención, dada la direccionalidad en la obtención de la información es prolectivo, ya que la obtención de esta se realizó simultáneamente a la ocurrencia de la maniobra.

El estudio se llevó a cabo en una unidad de medicina familiar (UMF) ubicada en el Estado de México, los participantes fueron cuidadores familiares de adultos mayores que acudieron a consulta y que se inscribieron al curso enfocado al cuidado del cuidador. Los criterios de inclusión fueron adultos de 20 a 59 años que supieran leer y escribir, que tuvieran a su cuidado un adulto mayor con el cual existe una relación de parentesco hasta la $3^{\text {a }}$ generación. Para la determinación de la muestra se utilizó la fórmula para poblaciones infinitas, quedó constituida por un grupo de 70 cuidadores, de los cuales solo uno abandonó la intervención.

La intervención fue denominada Cuidándose, el cual es un programa sobre las estrategias de cómo el cuidador familiar puede mejorar los procesos de afrontamiento y adaptación a este rol. El número de sesiones fueron 10, seis enfocadas al cuidado del cuidador familiar y cuatro al adulto mayor, de dos horas cada una, dos veces por semana; los temas impartidos fueron: información al cuidador familiar con relación al envejecimiento, la dependencia, el autocuidado, el continuar sus actividades de recreación, salud personal, ponerse en los zapatos del otro, escucha activa, manejo de conflictos, cuidados al AM relacionados con nutrición, eliminación, descanso, sueño, movilización, entorno seguro y, signos y síntomas de alarma; estos temas fueron el resultado del análisis de la estructura teórico conceptual y empírica de la psicoeducación de enfermería para el proceso de afrontamiento y adaptación al rol de cuidador familiar del adulto mayor. La entrega de la intervención estuvo a cargo de cinco facilitadores.

Los instrumentos de recolección de datos aplicados fueron: ficha de datos sociodemográficos que constó de nombre, número de seguridad social, teléfono de contacto, correo electrónico, edad, género, escolaridad, religión, ocupación, trabajo, estado civil, tiempo dedicado al cuidado y ocio, entre otros; esto con el fin de caracterizar al cuidador familiar del adulto mayor.

Se aplicó la Escala para medir el Proceso de Afrontamiento y Adaptación (ECAPS) ${ }^{11-18}$; la cual se fundamenta en el modelo conceptual de Callista Roy, el ECAPS demostró una alta validez y confiabilidad 
(alfa de Cronbach: 0.88, Rho de Spearman: 0.94); esta última determinada mediante la estabilidad de la escala a través del tiempo (prueba-reprueba) ${ }^{11-18}$.

El ECAPS tiene una medición ordinal, está conformado por 47 ítems, agrupados en cinco estilos y estrategias denominadas por Roy como factores, con una escala tipo Likert con cuatro puntajes de respuesta: nunca $=1$, algunas veces $=2$, casi siempre $=3$, siempre $=4$; cada item es una frase corta acerca de cómo una persona responde cuando experimenta una crisis o un evento difícil. Cabe destacar como consideración especial, que el afrontamiento en sí mismo es un indicador de cierto nivel de adaptación independientemente de las respuestas que se deriven de la actuación, por lo cual todas las personas afrontan y su respuesta frente a una situación parte de uno (1) y no de cero (o) 11-18. Los puntos de corte se establecieron a partir de obtener la mediana del puntaje (50\%) entre el rango de 47 a 188 para el total de la escala; se operacionaliza la baja capacidad de afrontamiento y adaptación con un puntaje de 47 a 117, y alta entre 118 y 188.

También se aplicó el Inventario de Habilidad de Cuidado (CAI por sus siglas en inglés) con el objetivo de medir la habilidad de cuidado para con el AM en tres dimensiones: conocimiento, valor y paciencia. El conocimiento implica tener el entendimiento y la razón para realizar las actividades de cuidado al AM, lo que refuerza su bienestar; el valor es la sensibilidad a estar abierto a las necesidades del presente y se obtiene de experiencias del pasado; la paciencia es dar tiempo y espacio para la expresión e incluye el grado de tolerancia ante la desorganización y problemas que limitan el crecimiento de sí mismo y de otros. El inventario consta de 37 ítems con escala tipo Likert, los puntajes de respuesta van de 1 a 7 puntos; se formulan ítems de forma positiva y negativa; los puntajes más altos indican mayor grado de cuidado para los ítems formulados de manera positiva; para los ítems negativamente formulados el puntaje se invierte. La dimensión de conocimiento consiste en 14 ítems, el de valor 13 ítems y la paciencia tienen 10 ítems. Con un puntaje de 37 a 259, la confiabilidad del instrumento fue de 0.89 y la validez de 0.80 , el nivel de habilidad de cuidado se clasifica como alto de 220 a 259 puntos, habilidad media de 155 a 219 y baja de 37 a $154^{19-25}$.

Por otra parte, el procesamiento de los datos se realizó mediante el Statistical Package for the Social Sciences (SPSS), versión 21 para IOS; con la base de datos completa se utilizó estadística descriptiva. El tratamiento estadístico se realizó con el fin de medir si la intervención Cuidándose generó alguna diferencia entre la evaluación pre y post; para los instrumentos ECAPS e Inventario habilidad de cuidado se consideraron los puntajes globales de respuesta de ambos instrumentos.

En relación a los aspectos éticos, este se trató de un estudio sin riesgo al brindar intervenciones inocuas, se tomó en consideración la Ley General de Salud ${ }^{26}$, en el capítulo único en materia de investigación en salud, al solicitar el consentimiento informado al inicio de la maniobra. El estudio contó con el dictamen favorable por el Comité de Investigación de la División de Ciencias de la Salud e Ingenierías de la Universidad de Guanajuato Campus Celaya- Salvatierra, con el registro CIDSC-3010504, así como con la autorización del Comité Local de Bioética número 15CHBo6220151023 del Instituto Mexicano del Seguro Social del Estado de México.

\section{RESULTADOS}

El grupo de estudio estuvo conformado por 69 cuidadores familiares del AM de 20 a 59 años, con una media de 50.6, una desviación estándar de 7.4, la mayor parte de los participantes fueron del género femenino con un 92.8\%; $42.0 \%$ son casados, el 33.3\% solteros y el $11.6 \%$ divorciados, $52.2 \%$ convive con su pareja. (Tabla 1) 
Con relación a la escolaridad, el $34.8 \%$ cuenta con licenciatura o posgrado, el $34.8 \%$ con bachillerato y el 30.4\% con primaria o secundaria. En relación a la ocupación, el 46.4\% se dedica a labores del hogar, seguido del $43.5 \%$ que realiza alguna actividad productiva y sólo el 10.1\% es pensionado. Dos terceras partes de este grupo (65.2\%) antes de dedicarse al cuidado del adulto mayor realizaban actividades productivas.

Tabla 1. Características sociodemográficas del cuidador familiar del adulto mayor $(n=69)$

\begin{tabular}{lrc} 
Variable & $f$ & $\%$ \\
\hline Género & & \\
\hline Femenino & 64 & 92.8 \\
\hline Masculino & 5 & 7.2 \\
\hline Estado civil & 23 & \\
\hline Soltera & $29 / 2$ & 44.3 \\
\hline Casada/ unión libre & $3 / 4 / 8$ & 21.8 \\
\hline Viuda/ Separada/ Divorciada & & \\
\hline Convive con su pareja & 36 & 52.2 \\
\hline Si & 33 & 47.8 \\
\hline No & &
\end{tabular}

Los días que brinda cuidados el sujeto de estudio al adulto mayor van de 6 a 7 días con el 50.7\%, seguido de 4 a 5 días el 11.6\%; el número de horas dedicadas a cuidar al AM tiene una media de 9.4 horas, con una desviación estándar de 3.4 con un rango mínimo de 3 y un máximo de 24 horas. Cabe señalar que las cinco principales tareas a las que se dedicaba el cuidador familiar cuando brindaba cuidados al AM fueron: 1) que los acompañaban a sus citas médicas (10.5\%),2) les preparaban sus alimentos (10.3\%), 3) les ayudaban en el control de los medicamentos (10.0\%), 4) les ayudaban a comer (8.4\%) y 5) realizaban actividades domésticas diversas (7.6\%);

Con respecto al tiempo que se lleva cuidando al AM, el $76.8 \%$ va de los 6 meses a más de 8 años; el 82.6\% de los cuidadores del adulto mayor práctica alguna religión, el 75.4\% es católico; el 75.4\% refirió realizar alguna actividad de ocio y recreación, de estos el 29.0\% la lleva a cabo de 2 a 3 días a la semana. Una cuarta parte de los cuidadores (24.6\%) no realiza actividades para su distracción.

Los resultados de la evaluación de la capacidad de afrontamiento y adaptación de los cuidadores familiares del adulto mayor previos a la intervención, mostraron que 68.1\% se clasificaron con baja capacidad de afrontamiento y adaptación a ser cuidador; y 31.9\% restantes tuvo una alta capacidad. Después de realizar la intervención psicoeducativa de enfermería Cuidándose, 82.6 \% calificó con una alta capacidad y sólo el 17.4 \% con baja capacidad. Al realizar una comparación entre la aplicación inicial y final, se evidencia un incremento de 50.7\% en la alta capacidad para ser cuidador familiar del AM. (Gráfico 1)

Los resultados del Inventario de la Habilidad de Cuidado de cuidadores familiares del adulto mayor previo a la intervención, mostraron que el 49.3\% tenían mediana habilidad de cuidado y $42 \%$ alta habilidad de cuidado; en la dimensión de conocimiento 52.2\% presentaron habilidad media; el 50.7\% obtuvieron clasificación de valor medio y en la dimensión de paciencia el 47.8\% presentaron un puntaje alto. Después de realizar la intervención psicoeducativa de enfermería Cuidándose, $52.2 \%$ se clasificaron con alta habilidad de cuidado, en la dimensión de conocimiento $52.2 \%$ tuvo una 


\section{Gráfica 1. Capacidad global de Afrontamiento y Adaptación del rol del cuidador familiar del AM antes y después de la intervención $(n=69)$}

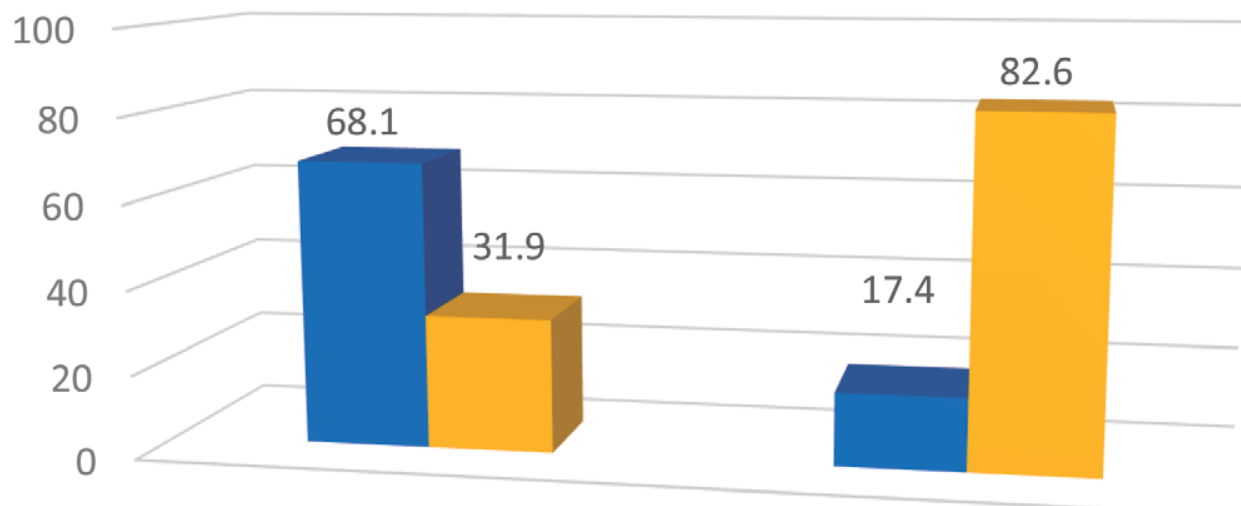

Antes

\section{Después}

Baja capacidad $\square$ Alta capacidad

Prueba de $\mathrm{Chi}^{2} \mathrm{Mc}$ Nemar $\mathrm{X}^{2}=30.29 ; \mathrm{gl}=1, \mathrm{p}=0.00001$

Gráfica 2. Puntaje global del inventario de habilidad de cuidado del rol del cuidador familiar del AM medición antes y después de la intervención $(n=69)$

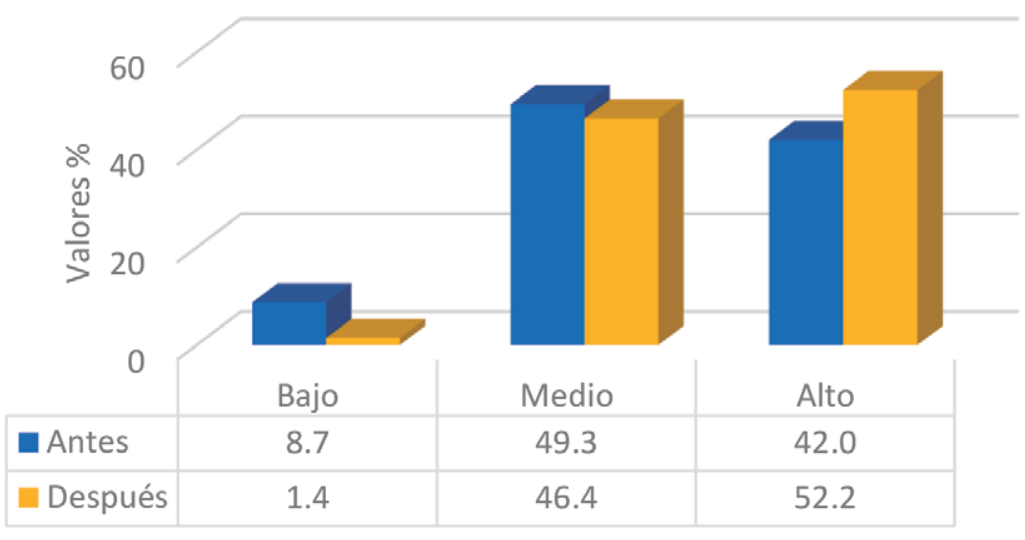

Prueba de $\mathrm{Chi}^{2}=6.125 ; \mathrm{gl}=1, \mathrm{p}=0.01$

habilidad alta, el 46.4\% habilidad media; en la dimensión de valor 52.2\% manifestó habilidad media; en la dimensión de paciencia el 56.5\% obtuvo una habilidad alta.

Al realizar una comparación entre la aplicación del antes y el después de la intervención psicoeducativa de enfermería Cuidándose a los cuidadores familiares del adulto mayor, se puede decir que el puntaje global del Inventario de Habilidad de Cuidado aumentó 10.2\% en el nivel alto. (Gráfico 2). Analizado por dimensiones en la de conocimiento alto incrementó $13.1 \%$, en la dimensión de valor y paciencia alto aumentó un $8.7 \%$ respectivamente. 


\section{DISCUSIÓN}

El grupo de estudio comparte características que señalan otras investigaciones sobre cuidadores familiares, es decir, en general los cuidadores son mujeres, adultas y casadas 3,23,27,28; en este caso, la mayoría de las participantes tuvieron estudios de nivel medio y superior; además un porcentaje mayor al 40\% realizan actividades productivas. Las características antes mencionadas pueden ser benéficas para cumplir con el rol de cuidador, pues un mejor nivel de escolaridad les permite captar en mayor medida las instrucciones para cumplir su función y el trabajo les permite cambiar de ambiente y no sólo limitarse a la atención del AM. No obstante; también podría implicar mayor carga y estrés al tener que cumplir con la actividad laboral y la atención de su familiar.

En el estudio realizado por Pérez-Jiménez ${ }^{28}$ refiere que el cuidador familiar puede cuidar hasta 12 horas diarias, situación que concuerda con esta investigación ya que se detectó que el número de horas dedicadas a cuidar al AM tiene una media de 9.6 horas; en el presente trabajo destaca, que más de la mitad del grupo brinda cuidados al AM casi toda la semana (de 6 a 7 días). En cuanto al aspecto positivo de esta dedicación; se ha señalado que la relación de parentesco favorece el deseo que el cuidador familiar tiene de estar con el AM para ayudarlo a realizar sus Actividades Básicas de la Vida Diaria (ABVD) y Actividades Instrumentales de la Vida Diaria (AIVD), situaciones que pueden influir en su capacidad para adaptarse al rol de cuidador ${ }^{29,30}$.

Las tareas que principalmente realiza el cuidador familiar fueron: acompañamiento a las citas médicas, preparación de alimentos, control de los medicamentos, ayuda para que los AM puedan comer y la realización de actividades domésticas diversas; esta situación coincide con el estudio de Flores-G E et al. ${ }^{7}$ y el de Manrique-Espinoza et al..$^{8}$ en relación con la pérdida de autonomía como la principal causa de necesidad de apoyo de los adultos mayores.

Conforme al objetivo del estudio, puede plantearse que la intervención psicoeducativa de enfermería Cuidándose, dirigida a los cuidadores familiares del AM, tuvo un efecto positivo ya que la capacidad de afrontamiento y adaptación al rol mostró un incremento notable. Por ejemplo, antes de la intervención sólo uno de cada tres cuidadores tenía alta capacidad, en cambio, después de la intervención cuatro de cada cinco quedaron calificados con alta capacidad de afrontamiento y adaptación.

La mayoría de los estudios consultados ${ }^{28,32-34}$ en que evalúan si existe afrontamiento y adaptación, destacan que los sujetos utilizan los estilos de afrontamiento enfocados a la resolución de problemas de manera racional, lo que pudiera traducirse en un tipo de afrontamiento que va de mediana a baja capacidad, sin embargo; en estos no se realizó intervención alguna, con el fin de mejorar la situación de los cuidadores; por lo que la intervención psicoeducactiva Cuidándose puede ser punto de partida para realizar actividades que apoyen un mejor afrontamiento y fomenten la adaptación al rol del cuidador familiar del adulto mayor. Aunado a esto, hasta ahora los esfuerzos se han enfocado en identificar el problema del proceso de afrontamiento y adaptación, este reconocimiento, aunque valioso sólo es el primer paso, lo siguiente es implementar intervenciones de enfermería y realizar una evaluación holística.

El contraste entre el nivel de la habilidad de cuidado previo a la intervención con el obtenido después de esta, permite afirmar que hubo mejoría, y aunque en las tres dimensiones consideradas hubo aumento, este se presentó en mayor medida en la dimensión de conocimiento, lo que podría indicar que los contenidos de la intervención fueron específicos para comprender mejor las condiciones del AM y de los cuidados necesarios más pertinentes, aunque debe considerarse que es más sencillo modificar niveles de conocimiento que aspectos actitudinales. Esta situación concuerda con 
otro estudio en el que también se incluyó una actividad de intervención, se reportaron cambios en el nivel de conocimientos y en paciencia, pero no así en la dimensión de valor ${ }^{31}$.

\section{CONCLUSIONES}

La intervención psicoeducativa Cuidándose, de acuerdo con los instrumentos que permitieron su evaluación, evidenciaron un aumento importante en la capacidad de afrontamiento y adaptación para mejorar el rol de cuidador; en cambio, en la evaluación de la habilidad de cuidado, se obtuvo un incremento moderado.

Estos resultados plantean la necesidad de realizar otras evaluaciones dirigidas a identificar las secciones que respondieron en mayor medida a las necesidades de los cuidadores y aquellas que deban reforzarse. Por otra parte, tendría que evaluarse esta misma intervención con grupos de adultos mayores con diferentes grados de dependencia; también, con cuidadores con otros niveles de escolaridad ya que el grupo estudiado tenía nivel superior al básico, lo que posibilita que las estrategias o estilos de afrontamiento puedan verse influidas por la escolaridad. Así mismo, es necesario investigar otro tipo de intervenciones o aspectos adicionales a la actual, dirigidas a favorecer cambios actitudinales.

Indudablemente, enfermería debe continuar con estos esfuerzos de probar diversas intervenciones que favorezcan la prevención, mantenimiento y recuperación de la salud, o como en el presente estudio, favorecer que la atención en esta etapa de la vida se realice con una mejor comprensión de sus necesidades y problemas, en la que se propicie mayor participación de la familia pero que esto no implique a su vez el deterioro de la salud de otros miembros del grupo.

\section{RESPONSABILIDADES ÉTICAS}

Protección de personas y animales. Los autores declaran que para esta investigación no se han realizado experimentos en seres humanos ni en animales.

Confidencialidad de los datos. En este artículo no aparecen datos confidenciales de los cuidadores familiares.

Derecho a la privacidad y consentimiento informado. Se solicitó el consentimiento informado previo al inicio de la intervención conforme lo marca el reglamento en materia de investigación en salud.

Financiamiento. No se recibió ningún tipo de financiamiento para la presente investigación.

Conflicto de intereses. Los autores declaran no tener conflicto de intereses.

Agradecimientos. A los cuidadores familiares que participaron en el estudio y a los profesores del Doctorado que fueron guía en la realización esta investigación.

\section{REFERENCIAS}

1. Medina-Chávez JH. Envejecimiento de la población y necesidad de la intervención interdisciplinaria. Rev Enferm Inst Mex Seguro Soc. 2015; 23(1):1-2. http://bit.ly/2mBcDqm

2. Fondo de Población de las Naciones Unidas. Población. Una población en crecimiento. New York: FPNU; 2019. http://bit.ly/2kIZRpi

3. Instituto Nacional de Estadística y Geografía. Esperanza de vida. México: INEGI; 2017. http://bit.ly/2kqgsOh

4. Alvarado-García MA. Adquiriendo habilidad en el cuidado: de la incertidumbre al nuevo compromiso. Aquichan. 2007; 7 (1): 25-36. http://bit.ly/2mqBs8k 
5. Borghi AC, De Castro VC, Marcon SS, Carreira L. Sobrecarga de familiares cuidadores de ancianos con la enfermedad de Alzheimer: un estudio comparativo Rev. Lat-Am. Enferm. 2013; 21(4): [08 pantallas]. http://bit.ly/2lZwaOS

6. Vega-Angarita OM. Percepción del apoyo social funcional en cuidadores familiares de enfermos crónicos. Aquichan. 2011; 11 (3): 274-86. https://doi.org/10.5294/aqui.2011.11.3.3

7. Flores GE, Rivas RE, Seguel PF. Nivel de sobrecarga en el desempeño del rol del cuidador familiar del adulto mayor, con dependencia severa. Cienc y Enferm. 2012; 18(1): 29-41. http://dx.doi.org/10.4067/So717-95532012000100004

8. Manrique-Espinoza B, Salinas-Rodríguez A, Moreno-Tamayo KM, Acosta-Castillo I, Sosa-Ortiz AL, Gutiérrez-Robledo LM, et al. Condiciones de salud y estado funcional de los adultos mayores en México. Salud pública Méx 2013; 55 (supl 2) S323-31. http://bit.ly/2kJ2J5y

9. González-González C, Sánchez-García S, Juárez-Cedillo T, Rosas-Carrasco O, Gutiérrez-Robledo LM, García-Peña C. Health care utilization in the elderly Mexican population: expenditures and determinants. BMC Public Health. 2011;11(1):192-202. https://doi.org/10.1186/1471-2458-11-192

10. Cuevas-Cancino JJ, Moreno-Pérez NE. Psicoeducación: intervención de enfermería para el cuidado de la familia en su rol de cuidadora. Enferm Univ. 2017; 14(3): 207-18.

http://dx.doi.org/10.1016/j.reu.2017.06.003

11. Roy C, Andrews H. The Roy adaptation model. za ed. USA: Pearson education; 2009.

12. Roy C, Chayput P. Coping and Adaptation processing scale-English and Thai version. Roy Adaptation Association Bulletin 2004; 6(2): 4-6.

13. Gutiérrez-López C. Veloza-Gómez MM, Moreno-Fergusson ME. Duran-de Villalobos MM, Lópezde Mesa C, Crespo O. Validez y confiabilidad de la versión en español del instrumento, escala de medición del proceso de afrontamiento y adaptación de Callista Roy. Aquichan. 2007; 7(1):54-63. http://bit.ly/2kVabu7

14. Lazcano-Ortiz M, Salazar-González BC, Gómez-Meza MV. Validación del instrumento: afrontamiento y proceso de adaptación de Roy en pacientes con diabetes mellitus tipo 2. Aquichan. 2008; 8(1): 116-25. http://bit.ly/2kpHvJx

15. Gutiérrez López, C. Escala de medición del proceso de afrontamiento y adaptación de Callista Roy: una propuesta metodológica para su interpretación. Hallazgos 2009; 6(12):201-13. http://bit.ly/2m2uHcM

16. Botero J, Sarmiento P. Validez de constructo de la escala de medición del proceso de afrontamiento y adaptación de Roy versión en español. Cultura del Cuidado Enfermería. 2011;1(1): 88-91. http://dx.doi.org/10.4321/S1132-12962013000300009

17. Callista C, Bakan G, Li Z, Nguyen TH. Coping measurement: Creating short form of Coping and Adaptation Processing Scale using item response theory and patients dealing with chronic and acute health conditions. Appl Nurs Res. 2016; 32 (11): 73-9. https://doi.org/10.1016/j.apnr.2016.06.002

18. Cunha M, Duarte J, Cardoso A, Ramos A, Quintais D, Monteiro R, et al. Caregiver skills inventory: factorial structure in a sample of portuguese participants. Millenium 2018; 2(6): 41-55. https://doi.org/10.29352/millo206.04.00164

19. Carreño-Moreno SP, Chaparro-Díaz L. Agrupaciones de cuidadores familiares en Colombia: perfil, habilidad de cuidado y sobrecarga. Pensam Psicol. 2017; 15(1):87-101.

http://dx.doi.org/10.11144/Javerianacali.PPSI15-1.ACFC 
20. Torres-Pinto X, Carreño-Moreno S, Chaparro-Díaz L. Factores que influencian la habilidad y sobrecarga del cuidador familiar del enfermo crónico. Rev Univ Ind Santander Salud. 2017; 49(2): 330-8.

http://dx.doi.org/10.18273/revsal.v49n2-2017006

21. Carreño-Moreno S, Barreto-Osorio RV, Duran-Parra M, Támara-Ortiz V, Romero E. Habilidad de cuidado y sobrecarga en cuidadores familiares de personas con enfermedad crónica. Rev Cubana Enfermer 2016; 32(3): 353-63. http://bit.ly/2kJ1Zxj

22. Carrillo-González GM, Sánchez-Herrera B, Barrera-Ortiz L. Habilidad de cuidado de cuidadores familiares de niños y adultos con enfermedad crónica. Índex enferm 2014; 23(3): 129-33. http://dx.doi.org/10.4321/S1132-12962014000200003

23. Tizoc-Marquez A, Higuera-Sainz JL, Uzueta-Figueroa MC, Peraza-Garay F, Medrano-Gil S. Nivel de habilidad del cuidador familiar en el cuidado a pacientes con enfermedad renal crónica del servicio de la UNEME del Hospital General de Culiacán Sin. Rev Med UAS 2012; 3(2): 56-64. http://bit.ly/2kTfn1K

24. Nkongho NO. The Caring Ability Inventory. En: Strickland OL, Dilorio C, (eds). Measurement of Nursing Outcomes (Vol 3). 2. ed. New York: Springer Publishing Company; 2003, 184-98.

25. Bustos-Azúcar S, Rivera-Fuentes N, Pérez-Villalobos C. Habilidad de cuidado en estudiantes de enfermería de una universidad tradicional de Chile. Cienc enferm 2016; 22(2): 117-27. http://dx.doi.org/10.4067/So717-95532016000200009

26. Secretaría de Salud. Reglamento de la ley general de salud en materia de investigación para la salud. Última Reforma. México: SS; 24-03-2014. http://bit.ly/2kJgDrv

27. Joling KJ, Van-Marwijk HWJ, Smit F, van der Horst HE, Scheltens P, van de Ven PM, et al. Does a family meetings intervention prevent de pression and anxiety in family caregivers of dementia patients? A randomized trial. PLoS ONE. 2012; 7(1): e30936. https://doi.org/10.1371/journal.pone.0030936

28. Pérez-Jiménez D, Rodríguez-Salvá A, Herrera-Travieso DM, García-Roche R, Echemendía-Tocabens B, Chang-de la Rosa M. Caracterización de la sobrecarga y los estilos de afrontamiento en el cuidador informal de pacientes dependientes. Rev Cubana Hig Epidemiol, 2013; 51(2): 174-83. http://bit.ly/2mqOqLy

29. Roy C. Research based on the Roy Adaptation Model: Last 25 years. Nurs Sci O. 2011; 24 (4) 312-20. https://doi.org/10.1177/0894318411419218

30. Roy C. Generating Middle Range Theory, From evidence to practice. New York: Springer Publishing Company; 2013.

31. De la Huerta R, Corona J, Méndez J. Evaluación de los estilos de afrontamiento en cuidadores primarios de niños con cáncer. Rev Neurol Neurocir Psiquiat 2006; 39 (1): 46-51. http://bit.ly/2kJmzO2

32. Aldana G, Guarino L. Sobrecarga, afrontamiento y salud en cuidadoras de pacientes con demencia tipo Alzheimer. Summa psicol UST. 2012; 9(1): 5-14. http://bit.ly/2m5fgOL

33. Cedillo-Torres AG, Grijalva MG, Santaella-Hidalgo GB, Cuevas-Abad M, González-Pedraza A. Asociación entre ansiedad y estrategias de afrontamiento del cuidador primario de pacientes postrados. Rev Med Inst Mex Seguro Soc. 2015; 53(3): 362-7. http://bit.ly/2kv7jnM

34. Díaz-Álvarez JC, Rojas-Martínez MV. Cuidando al cuidador: efectos de un programa educativo. Aquichán 2009; 9(1): 73-92. http://bit.ly/2m4pz7D 